\title{
Brain homogenate decoys for antigen-specific cell
}

\section{amplification}

J. Daniel Griffin ${ }^{1,2}$, Sebastian G. Huayamares ${ }^{1}$, Towne R. Walston ${ }^{3}$, Jimmy Y. Song ${ }^{2}$, Michael Shao $^{4}$, Alexander R. Sedlacek ${ }^{4}$, Deanna L. Diaz ${ }^{4}$, Aparna R. Chakravarti ${ }^{1}$, and Cory J.

Berkland ${ }^{1,2,4, *}$

${ }^{1}$ Bioengineering Graduate Program, University of Kansas, Lawrence, KS

${ }^{2}$ Department of Pharmaceutical Chemistry, University of Kansas, Lawrence, KS

${ }^{3}$ School of Medicine, University of Kansas Medical Center, Kansas City, KS

${ }^{4}$ Department of Chemical and Petroleum Engineering, University of Kansas, Lawrence, KS

*Corresponding author: berkland@ku.edu; 2030 Becker Drive, Room 320E, Lawrence, KS 66047

Keywords: Autoimmunity, Biomaterial, Immunotherapy, Experimental Autoimmune Encephalomyelitis (EAE), Antigen-Specific Immune Decoy 
Materials. Gelatin type A from porcine skin, methacrylic anhydride, and Irgacure 2959 were purchased Sigma Aldrich (St. Charles, MO). Mouse brain material was obtained from SJL/J mice (Envigo, Indianapolis, IN) housed in specified, pathogen free conditions at the University of Kansas. Mouse brain homogenate was prepared adding 1X PBS to obtain a concentration of 1 $\mathrm{g} / \mathrm{mL}$. Using a sonicator probe, the mixture was homogenized for 2 minutes using 10 seconds on, 2 seconds off regimen. The resulting mouse brain homogenate was stored at $-20^{\circ} \mathrm{C}$. 2,5 dioxopyrrolidin-1-yl 1-azido-3,6,9,12-tetraoxapentadecan-15-oate (azido-PEG4-NHS Ester) was purchased from Click Chemistry Tools (Scottsdale, AZ). Tris(3hydroxypropyltriazolylmethyl)amine (THPTA), and sodium ascorbate (NaAsc) were purchased from Sigma-Aldrich (St. Louis, MO). Copper (II) sulfate pentahydrate $\left(\mathrm{CuSO}_{4} \cdot 5 \mathrm{H}_{2} \mathrm{O}\right)$ was purchased from Acros Organics (Geel, Belgium). Alkyne-functionalized PLP with an $N$-terminal 4-pentynoic acid (homopropargyl, hp) modification, hpPLP ${ }_{139-151}$ (hp-HSLGKWLGHPDKF-OH) was purchased from Biomatik (Cambridge, ON, Canada). Unmodified PLP $139-151$ (NH2HSLGKWLGHPDKF-OH) used for EAE induction, rechallenge assays, and anti-PLP IgG immunostaining was purchased from PolyPeptide Laboratories (San Diego, CA). Incomplete Freund's adjuvant (IFA) and killed Mycobacterium tuberculosis strain H37RA were purchased from Difco (Sparks, MD). Pertussis toxin was purchased from List Biological Laboratories (Campbell, CA). PE/Cy7-conjugated anti-mouse CD3, PE-conjugated anti-mouse CD86, Alexa Fluor 647-conjugated anti-mouse CD19, and Brilliant Violet 421-conjugated anti-mouse CD11c were purchased from BioLegend (San Diego, CA). All other chemicals and reagents were analytical grade and used as received.

Preparation of Gelatin Methacrylate. A 10\% (w/v) solution of porcine gelatin was prepared in PBS. The solution was warmed to $60^{\circ} \mathrm{C}$ and stirred vigorously as methacrylic 
anhydride was added dropwise (up to $8 \%$ of total gelatin content) to methacrylate the amine groups on gelatin polymers. After two hours of continued mixing, the solution was diluted with PBS and dialyzed (12-14 kDa cutoff) with deionized water for one week with twice daily water changes. The dialyzed GelMA was frozen at $-80^{\circ} \mathrm{C}$ before being lyophilized to yield a purified solid product. Fabrication of Homogenate-GelMA Scaffolds. The mbhGelatin scaffolds were produced by creating a $2 \mathrm{X}(14 \%)$ solution of GelMA containing an excess $(0.02 \%)$ of Irgacure 2959 photocrosslinker and a 2X solution of mouse brain homogenate in 1X PBS that was proportional to GelMA content by weight. GelMA and homogenate-containing solutions were mixed in equal parts and added to wells of a 24-well plate. Using a UV light source, the mixtures were photocrosslinked for 7 minutes. Gelatin constructs were then frozen overnight at $-80^{\circ} \mathrm{C}$ and lyophilized for storage.

Synthesis of plpGelatin Materials. PLP-conjugated materials were prepared as previously described $^{1}$. Briefly, blank gelatin scaffolds were hydrated in $\mathrm{pH}$ 8.3, $50 \mathrm{mM}$ HEPES buffer. 2 $\mathrm{mg} / \mathrm{mL}$ azido-PEG4-NHS was added and the mixture was reacted for 4 hours at room temperature. The materials were washed 5 times in deionized water before being placed in a solution of 2 $\mathrm{mg} / \mathrm{mL}$ hpPLP ${ }_{139-151}$ prior to the addition of a premixed solution of $4.5 \mathrm{mM}$ THPTA and $0.8 \mathrm{mM}$ copper (II) sulfate pentahydrate. Sodium ascorbate $(16 \mathrm{mM})$ was added to commence the reaction, which was run overnight at room temperature. The resulting plpGelatin materials were washed 5 times in deionized water and stored in 100\% ethanol. PLP conjugation to collagen sponges was determined using a 20 minute Reverse-Phase HPLC method employing a 95/5 to 30/70 aqueous:organic gradient scheme on a C4 RP column.

Characterizing Complex Decoys. Scanning electron microscopy (SEM) was carried out at 150X, 500X, and 1000X magnifications to approximate gelatin pore size. SEM images were taken 
on an FEI Technai F20 XT Field Emission Transmission Electron Microscope with dehydrated gelatin hydrogels. mbhGelatin constructs of varying homogenate contents were subjected to rheological and mechanical testing to characterize homogenate effects on these properties. Rheological testing was carried out across oscillatory frequency and strain sweeps using an AR2000 rheometer. Elastic modulus, G', and loss modulus, G”, were recorded. Frequency sweeps consisted of oscillatory measurements at a low fixed strain (1\%) with increasing frequency amplitudes to determine the G' and G', of the constructs as a function of frequency. The strain sweeps consisted of oscillatory measurements at a fixed frequency $(1 \mathrm{~Hz})$ with increasing strain amplitudes. Tan $\delta$, or the loss factor, is the ratio of G', to $\mathrm{G}^{\prime}$ and is used in strain sweeps to help recognize the strain at which the gel's structure breaks mechanically. A release study was conducted by procuring $20 \mathrm{mg}$ sections of mbhGelatin at varied $\mathrm{G}: \mathrm{H}$ ratios and adding to scintillation vials containing $20 \mathrm{~mL}$ of PBS. Across time points, $1 \mathrm{~mL}$ of bulk solution was sampled and replaced with fresh PBS. Samples were quantified for overall protein content using a Pierce BCA Protein Assay Kit (ThermoFisher Scientific, Waltham, MA). The loss of released material from sampling was accounted for when calculating cumulative release over time.

Retained Antigen-Specificity Analysis. 2:1 mbhGelatin, Blank Gelatin, and PLP $139-151^{-}$ conjugated gelatin constructs were embedded and cryosectioned ( $20 \mu \mathrm{m}$ sections) onto microscope slides. Each sample was fixed using 10\% neutral buffered formalin and blocked with $1 \%$ bovine serum albumin. Samples were then incubated with mouse serum containing antibodies for PLP 139 151. Following the incubation, samples were washed, and bound antibody was detected using HRPconjugated anti IgG. TMB substrate was incubated with the slides for 15 minutes, and pictures were taken to visualize PLP-specific binding. 
Induction of EAE and Spleen Harvest. Experimental autoimmune encephalomyelitis was induced as previously described ${ }^{1}$. All animal studies were performed with authorization from the University's Institutional Animal Care and Use Committee. 4-6 week-old female mice were subcutaneously administered the PLP $_{139-151}$ autoantigen peptide epitope in complete Freund's Adjuvant $\left(200 \mu \mathrm{L}\right.$ of emulsion containing $1 \mathrm{mg} / \mathrm{mL}$ PLP $_{139-151}$ and $4 \mathrm{mg} / \mathrm{mL}$ Mycobacterium tuberculosis) with the intraperitoneal coadministration of pertussis toxin capsid (100 $\mu \mathrm{L}$ of 1 $\mathrm{ng} / \mu \mathrm{L})$. Intraperitoneal injection of pertussis toxin was repeated on day two post-induction. At peak of disease on day 12 of the study, splenocytes were harvested and isolated for use as previously described.

Ex Vivo Screening with EAE Splenocytes. At peak of disease (Day 12), mixed EAE splenocytes were harvested and plated at $1.5 \times 10^{6}$ cells per well $(500 \mu \mathrm{L})$ in an untreated 24 -well plate containing either media alone (-PLP), soluble antigen rechallenge (+PLP), mbhGelatin sponge, or Blank Gelatin sponge. After 96 hours, sponges were minced with microscissors and the culture suspension was collected and passed through a cell strainer for further processing and analysis.

Fluorescent Staining and Flow Cytometry. Cell samples were collected and washed in FACS buffer containing $1 \mathrm{X}$ PBS $+5 \%$ fetal bovine serum $+0.1 \%$ sodium azide. Samples were resuspended in a solution containing Zombie Aqua viability stain (Biolegend) and were incubated for 20 minutes at room temperature. Following the incubation, fluorescent antibodies were added at manufacturer recommended concentrations for 30 minutes at $4^{\circ} \mathrm{C}$. Samples were washed and run on a BD FACSFusion cytometer, where 100,000 events were collected per sample. Data were analyzed using FlowJo and GraphPad Prism. Gating schemes were identical to those previously described $^{1}$. 
Resazurin Cell Metabolism Assay. After 96 hours of incubation, isolated cell samples were incubated with $75 \mu \mathrm{M}$ resazurin (7-hydroxy-3H-phenoxazin-3-one 10-oxide) for 3 hours. Metabolic reductive capacity was measured by assessing changes in fluorescence (560 excitation, 590 emission, Spectramax M5, Molecular Devices, San Jose, CA). Background fluorescence was subtracted out by taking measurements of RPMI media alone.

Statistical Analysis. Statistical analysis was performed using an ordinary one-way ANOVA with uncorrected Fisher's LSD test. Statistical significance was set beginning at $p<0.05$ and was assessed by comparing treatment values one group as a control (unless otherwise stated). All analyses were performed using GraphPad Prism software (GraphPad Software, Inc., San Diego, CA). 

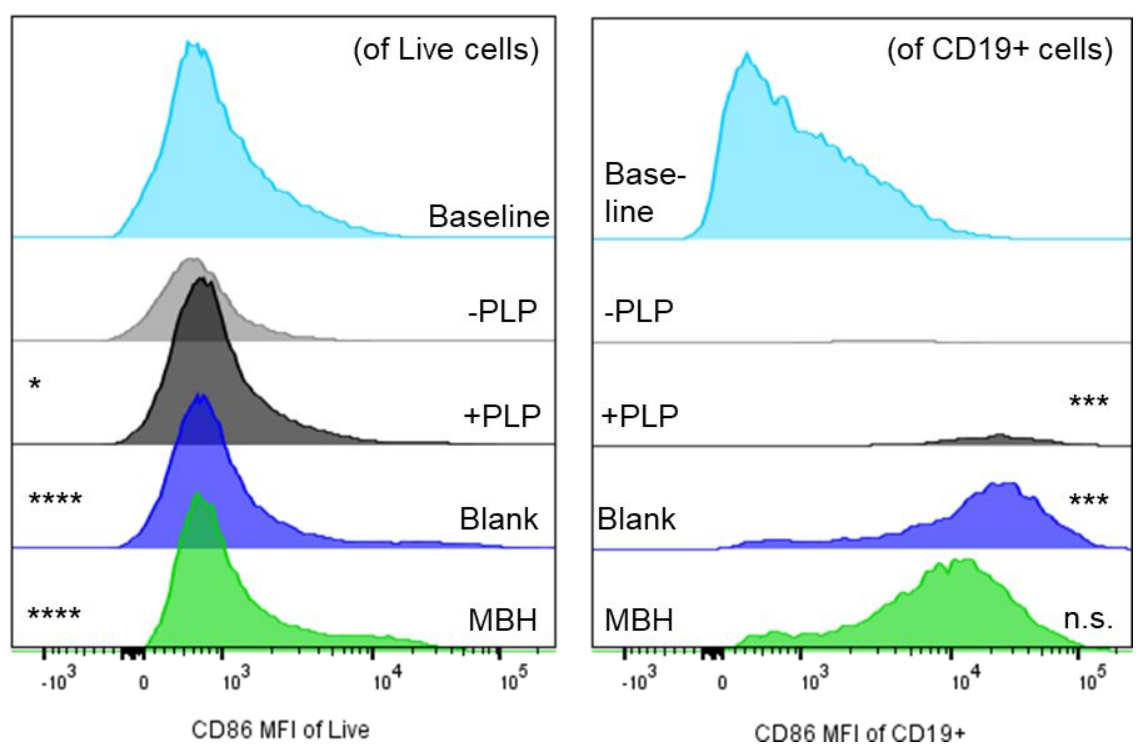

Figure S1. Histograms of CD86 expression as mean fluorescence intensity (MFI) among all live cells (left) and CD19+ cells (right). The increased prevalence of CD19+ cells after 96 hours for splenocytes incubated with mbhGelatin corroborated an increase in total CD86+ cells, though CD86 expression was not upregulated within this population. Baseline measurements at 0 hours are included for comparison to the 96 hour time point. The abundance of CD19+ cells expressing CD86 (albeit at lower levels than Blank Gelatin and +PLP splenocytes) seems to have promoted signal propagation for the expansion of PLP-specific B cells. (Statistical analysis was performed against -PLP as a control. $\mathrm{n}=3$ /group, n.s. - not significant, ${ }^{*} \mathrm{p}<0.05,{ }^{*} \mathrm{p}<0.01,{ }^{*} * \mathrm{p}<0.001$, $* * * * \mathrm{p}<0.0001)$. 
a. (Population gating)
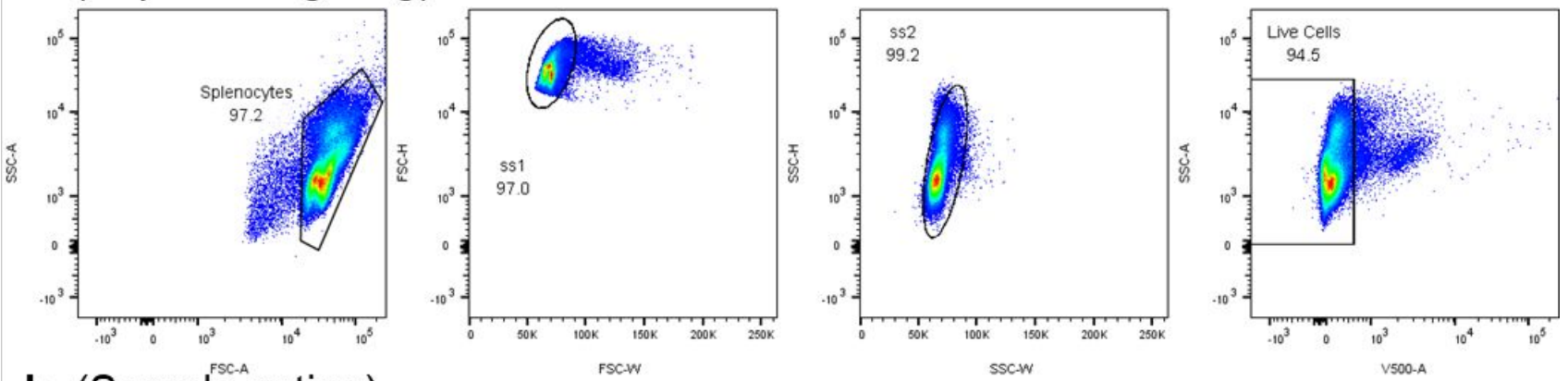

b. (Sample gating)
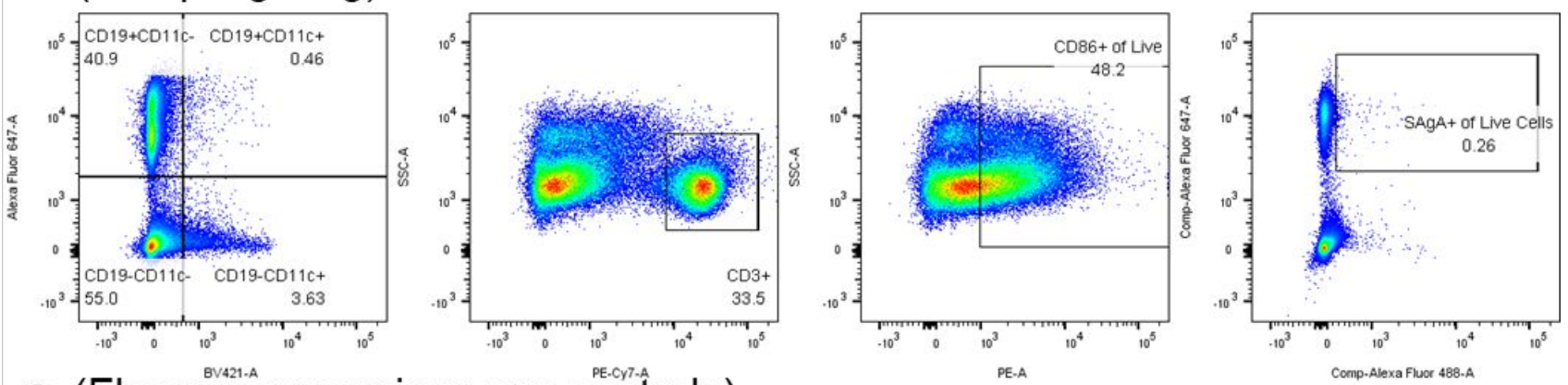

c. (Fluorescence minus one controls)
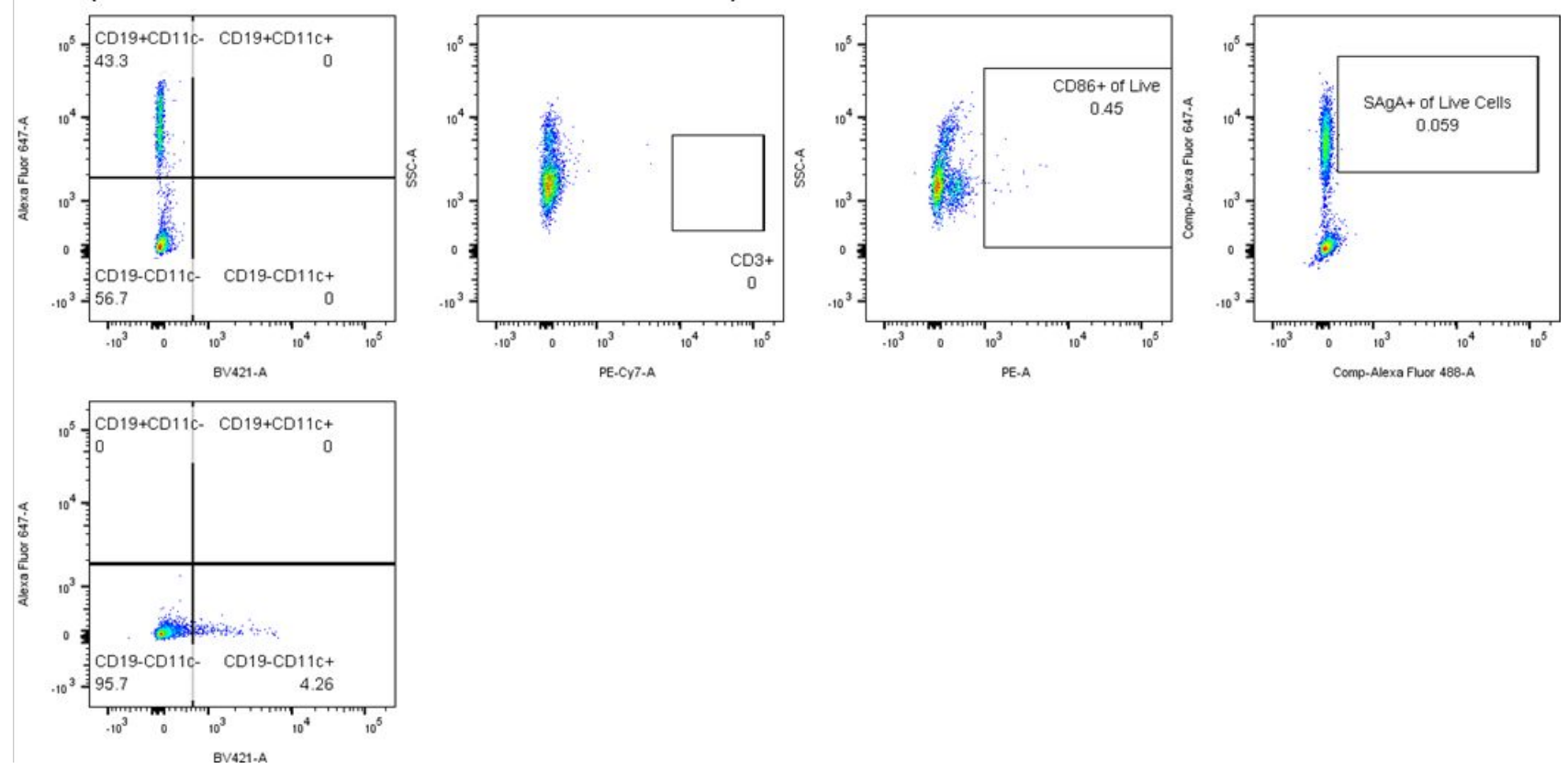

Figure S2. Gating strategies used for the flow cytometry studies are presented. a. Live cell gating was narrowed from left to right by selecting splenocytes from the SSC FSC plot (left), selecting for singlets using FSC-H FSC-W and SSC-H-SSC-W (middle left, middle right), and excluding cells staining positive for Zombie Aqua in the V500 channel (right). b. Gating strategies are presented for a stained sample, including CD19-A647 and CD11c-BV421 (left), CD3-PE/Cy7 (middle left), CD86-PE (middle-right), and SAgA-FITC (right). c. Corresponding fluorescence minus one (FMO) controls are presented beneath corresponding gating for the positively stained sample. 


\section{Reference}

1. Griffin, J. D.; Song, J. Y.; Huang, A.; Sedlacek, A. R.; Flannagan, K. L.; Berkland, C. J., Antigen-specific immune decoys intercept and exhaust autoimmunity to prevent disease. Biomaterials 2019, 222, 119440. 